\title{
POLÍTICAS PÚBLICAS DE EDUCAÇÃO EM SAÚDE PARA A PREVENÇÃO DE COMORBIDADES E DOENÇAS CARDIOVASCULARES
}

\section{PUBLIC HEALTH EDUCATION POLICIES FOR PREVENTION OF COMORBIDITIES AND CARDIOVASCULAR DISEASES}

\author{
Elisabeth de Almeida Figueiredo* \\ Daniella Cavalcante Antunes** \\ Maria Geralda de Miranda***
}

\begin{abstract}
Resumo: A obesidade tornou-se um dos mais expressivos problemas de saúde pública no mundo. O excesso de peso, geralmente, não é abordado, adequadamente, na infância e na adolescência e, com o passar do tempo, outras doenças se instalam ou se intensificam, são as comorbidades como diabetes mellitus, hipertensão arterial, dentre outras e as doenças cardiovasculares. Estima-se que, em 2015, 17,7 milhões de pessoas morreram por doenças cardiovasculares à nível global. Este artigo tem por objetivo levar à criação de políticas públicas de educação em saúde, com atendimento escolar específico e devido encaminhamento dos alunos portadores de obesidade às unidades de saúde. A educação alimentar, com mudança de hábitos e a atividade física regular devem ser estimuladas, como medidas preventivas e corretivas, da obesidade e de doenças cardiovasculares na fase precoce da vida.
\end{abstract}

Palavras-chave: Obesidade Infantil e na Adolescência. Educação Alimentar. Doenças Cardiovasculares.

Abstract: Obesity has become one of the most significant public health problems in the world. Overweight is generally not adequately addressed in childhood and adolescence and, over time, other diseases become established or intensify, such as diabetes mellitus, arterial hypertension, among others, and cardiovascular diseases. It is estimated that in 2015, 17.7 million people died from cardiovascular diseases globally. This article aims to lead to the creation of public health education policies, with specific school attendance and due to the referral of students with obesity to health units. Food education, with changing habits and regular physical activity

\footnotetext{
* Mestranda do Programa de Pós-graduação Profissional em Desenvolvimento Local do Centro Universitário Augusto Motta - UNISUAM. Pós-graduada em Gestão Ambiental e Desenvolvimento Sustentável pela UNINTER/PR. Pós-graduada em Saneamento Ambiental pelo Centro universitário Leonardo da Vinci/SC. Graduada em Segurança do Trabalho pela Universidade Estácio de Sá. Graduada em Nutrição pela UFRJ.

** Mestranda do Programa de Pós-graduação Profissional em Desenvolvimento Local do Centro Universitário Augusto Motta - UNISUAM. Pós-graduada em Psicomotricidade pela Universidade Estácio de Sá. Graduada em Fonoaudiologia pela Universidade Estácio de Sá.

***Pós-doutora em Políticas Públicas, Pesquisadora do Programa de Pós-graduação em Desenvolvimento Local, Centro Universitário Augusto Motta. E-mail: mgeraldamiranda@gmail.com.
} 
should be stimulated, as preventive and corrective measures, of obesity and cardiovascular diseases in the early phase of life.

Keywords: Child Obesity and Adolescence. Nutrition education. Cardiovascular Diseases. 


\section{INTRODUÇÃO}

Nas últimas décadas, tanto em países desenvolvidos como em países em desenvolvimento, a obesidade está sendo vista como uma doença crônica e epidêmica em crianças e adolescentes, "[...] sendo objeto de estudo de revisão que confirmou a atuação da gordura ectópica na liberação de adipocitinas, lipotoxinas e glicotoxinas que acarretam disfunções cardiovasculares." (COSTA et al., 2017, p. 211).

Para Oliveira e outros autores (2004, p. 239) "Além do risco aumentado da criança e do adolescente obeso permanecer neste estado quando adultos, [...] estudos longitudinais sugerem que o tempo de duração da obesidade está diretamente associado a morbimortalidade por doenças cardiovasculares".

A Lei 9.394, de 20 de dezembro de 1996, de Diretrizes e Bases da Educação Nacional (BRASIL, 1996), foi alterada, recentemente, pela Lei no 13.666, de 16 de maio de 2018, para acrescentar o tema transversal da educação alimentar e nutricional no currículo escolar (BRASIL, 2018a).

A Lei 9.394 passou a vigorar, após decorridos cento e oitenta dias da publicação oficial, acrescida do $\S 9^{\circ} \mathrm{A}$ - a educação alimentar e nutricional será incluída entre os temas transversais de que trata o caput (BRASIL, 1996). "[...] se apresenta a possibilidade de uma nova organização curricular, que permita eliminar a clássica divisão entre as disciplinas e introduzir novos arranjos de conteúdo, com base na interdisciplinaridade e temas transversais." (CARVALHO, 2012, p. 54).

O Projeto de Lei da Câmara no 102, de 14 de setembro de 2017, deu origem à proposta de incluir o tema Educação Alimentar e Nutricional no conteúdo das disciplinas de Ciências e Biologia dos currículos do ensino fundamental e do ensino médio, respectivamente (BRASIL, 2017). A justificação da iniciativa foi baseada no aumento da taxa de obesidade infantojuvenil.

A partir das premissas apresentadas, surge a proposta de uma prática educacional em saúde, considerando a circunstância favorável da recente inclusão do tema transversal Educação Alimentar e Nutricional, pela Lei no 13.666, no processo de aprendizagem do Ensino Fundamental, etapas finais, e do Ensino Médio (BRASIL, 
2018a). Destaca-se a urgência da inserção da abordagem prevencionista em relação à obesidade e às comorbidades (doenças que se instalam ou se intensificam com a obesidade; exemplos, diabetes mellitus, hipertensão arterial) e consequentes doenças cardiovasculares.

O artigo no 196 da Constituição da República Federal de 1988 dispõe que a saúde é direito de todos e dever do Estado, garantido mediante políticas sociais e econômicas que visem à redução do risco de doença, de outros agravos e ao acesso universal e igualitário às ações e serviços para sua promoção, proteção e recuperação (BRASIL, 1988).

O artigo no 205 da Constituição Federal e o artigo no 53 do Estatuto da Criança e do Adolescente, dispõem a todos a educação como direito (BRASIL, 1988; 1990). As mudanças nas Diretrizes Curriculares Nacionais para a Educação Básica ampliaram os direitos à educação de crianças e adolescentes. O Ministério da Educação apresentou um dos fundamentos do projeto de Nação:

[...] a formação escolar é o alicerce indispensável e condição primeira para o exercício pleno da cidadania e o acesso aos direitos sociais, econômicos, civis e políticos. A educação deve proporcionar o desenvolvimento humano na sua plenitude, em condições de liberdade e dignidade, respeitando e valorizando as diferenças (BRASIL, 2013a, p. 4).

De acordo com a Política Nacional de Promoção à Saúde (PNPS), o objetivo geral é o de promover a equidade e a melhoria das condições e dos modos de viver, ampliando a potencialidade da saúde individual e coletiva e reduzindo vulnerabilidades e riscos à saúde decorrentes dos determinantes sociais, econômicos, políticos, culturais e ambientais (BRASIL, 2014a).

Segundo Luz (2008, p. 353) "[...] o termo saúde designa, portanto, uma afirmação positiva da vida e um modo de existir harmônico, não incluindo, em seu horizonte, o universo da doença. [...] que saúde, em sua origem etimológica, é um estado positivo do viver $[\ldots] "$.

O Programa Nacional de Alimentação Escolar - PNAE é um programa de grande impacto social, considerado o mais abrangente dos programas voltados à alimentação e nutrição na América do Sul. Atualmente, são atendidos 37 milhões de 
alunos. O programa tem por objetivo a melhoria das necessidades nutricionais dos estudantes, durante o período em sala de aula (BRASIL, 2013b). A Lei no 11.947, de 16 de junho de 2009 dispõe sobre o atendimento da alimentação escolar e do Programa Dinheiro Direto na Escola aos alunos de Educação Básica (BRASIL, 2009). Determina que no mínimo $30 \%$ dos recursos sejam destinados para a aquisição de produtos da agricultura familiar. A Resolução FNDE (Fundo nacional de Desenvolvimento da Educação) no 26, de 17 de junho de 2013, entrou em vigor com a finalidade de melhorar o atendimento no âmbito do PNAE (BRASIL, 2013b).

\section{OBESIDADE}

Os estudos sobre obesidade no país têm explanado o tema na ótica da epidemiologia. Medidas específicas são avaliadas sob o impacto que oferecem (BRASIL, 2014b). "O mundo moderno se transformou em um ambiente com variáveis inesgotáveis de alimentos baratos, fartos, palatáveis e de alta densidade energética e de avanços tecnológicos, feitos para diminuir a atividade física." (POLSK, 2016, p. 775).

A obesidade vem aumentando, rapidamente, nas gerações mais recentes e a sua prevalência na sociedade contemporânea é um grande desafio na área de saúde pública. Segundo Polsky (2016, p. 775), um indivíduo desenvolve obesidade pela interação entre fatores genéticos, ambientais e comportamentais que agem no equilíbrio energético.

Somente nos últimos 15 anos, a obesidade assumiu prioridade nas políticas públicas, diante da sua magnitude e da associação com doenças crônicas não transmissíveis (DCNT), especialmente.

Estratégias que ultrapassem o âmbito de ação do setor saúde são necessárias, dadas as dificuldades em universalizar medidas individualizadas, como intervenções cirúrgicas, além dos limites que os próprios indivíduos enfrentam para modificar suas escolhas pessoais, alimentares ou de prática de atividade física, em contextos adversos à adoção de práticas saudáveis. (DIAS, 2017, p. 4). 
A obesidade é categorizada, na CID-11 para Estatísticas de Mortalidade e Morbidade (versão 2018) no capítulo 05 Doenças endócrinas, nutricionais e metabólicas"11 (WORLD HEALTH ORGANIZATION, 2018). A obesidade é mencionada como um dos fatores de risco e como doença.

O Instituto Brasileiro de Geografia e Estatística (2018), IBGE, divulgou os resultados da pesquisa de Orçamentos Familiares que destacam o aumento de indivíduos obesos no Brasil, ao longo dos anos.

A obesidade também causa transtornos na vida diária das pessoas acometidas. Os mais frequentes são problemas estéticos acompanhados da dificuldade em andar, na obtenção de roupas de tamanhos maiores, restrição nos cuidados higiênicos, problemas com as entradas de acesso, limitações quanto a assentos, atividades físicas, e, com frequência, ser alvo de bullying.

\section{HÁBITOS ALIMENTARES INADEQUADOS E SEDENTARISMO}

Segundo Oliveira e outros autores (2003, p.145):

As preferências alimentares das crianças, assim como as atividades físicas, são práticas influenciadas, diretamente, pelos comportamentos dos pais, que persistem, frequentemente, na vida adulta, o que reforça a hipótese de que os fatores ambientais são decisivos na manutenção ou não do peso saudável.

Há estudos sobre o comportamento dos adolescentes, em relação a alguma forma de tempo de tela, seja a visualização televisiva, jogos e internet, por horas consecutivas, diariamente, e o aumento do índice de massa corporal, IMC. Segundo Biddle, Bengoechea e Wiesner (2017, p. 13), "Em resumo, evidências de revisões sistemáticas sintetizando estudos observacionais transversais indicam uma associação entre visualização de TV, tempo de tela e adiposidade na juventude e algumas evidências, mas menos claras, para o uso de computadores" ${ }^{\prime \prime}$.

\footnotetext{
1 Título original: "ICD-11 for Mortality and Morbidity Statistics (2018 version). 05 Endocrine, nutritional or metabolic diseases"

2 Texto original: "In summary, evidence from systematic reviews synthesizing observational crosssectional studies indicates an association between TV viewing, screen time and adiposity in youth, and some evidence, but less clear, for the use of computers".
} 
"Sabe-se ainda que fatores comportamentais são adquiridos na infância e adolescência, tornando essencial seu estudo para identificação de hábitos saudáveis." (COELHO et al., 2015, p. 407).

Uma boa alimentação e nutrição são requisitos básicos para ter uma vida saudável, pois possibilitam o crescimento e o desenvolvimento humano com qualidade. Porém, não existe um padrão ou uma receita de alimentação saudável universal e que respeite as necessidades nutricionais de acordo com os atributos coletivos, específicos e individuais de todos. (COELHO et al., 2015, p. 410).

$\mathrm{O}$ poder e influência da mídia e a diversidade de produtos em suas embalagens coloridas influenciam os hábitos alimentares diários. Refeições congeladas, refrigerantes, balas, chicletes, biscoitos recheados, salgadinhos fritos, sucos industrializados adoçados, sorvetes cremosos, embutidos, bolos com cobertura são alguns tipos de alimentos ricos em açúcares, sal e conservantes.

No cotidiano alimentar das escolas sublinha-se a redução da prevalência da desnutrição, em termos gerais, no Brasil e aumento de frequência de sobrepeso e obesidade. Um problema de saúde decorrente de hábitos não saudáveis (FREITAS, 2013, p. 980).

A orientação é melhorar a qualidade da alimentação diária, consumindo alimentos saudáveis, ricos em nutrientes, de forma equilibrada.

A AHA (American Heart Association) publicou um guia "Gestão do estilo de vida para reduzir o risco cardiovascular"3 direcionado para o público adulto. (ECKEL et al., 2013).

Observa-se que ainda há uma lacuna em diretrizes e modelos multidisciplinares de práticas de prevenção de doenças cardiovasculares voltados para crianças e adolescentes.

\section{COMORBIDADES}

\footnotetext{
3 Título original: "Guideline of lifestyle management to reduce cardiovascular risk".
} 
A palavra comorbidade define a doença que se instala ou se intensifica com a obesidade, devido ao acúmulo de gordura no organismo e, simultaneamente, a elevação do peso e massa corporal. Como consequência, a ocorrência de hipertensão arterial, diabetes, dislipidemias e outras doenças, que são denominadas de comorbidades prognósticas da obesidade.

O Brasil está entre os 10 países com maior número de mortes causadas por doenças cardiovasculares, sendo a principal razão de morte em mulheres e homens no país.

"As doenças cardiovasculares (DCVs) são as principais causas de morte na população brasileira e são responsáveis por pelo menos $20 \%$ das mortes em nossa população com mais de 30 anos de idade" (MANSUR; FAVARATO, 2016, p.21).

A metade das causas de morte por DCV, antes dos 65 anos, pode ser atribuída à pobreza. Da mesma forma, baixa escolaridade contribui para pobreza, aumentando o índice de mortalidade por DCV. Alimentação inadequada, baixa atividade física, consumo de álcool e tabagismo são outros importantes fatores de risco para as DCVs, e é mais prevalente nas classes sociais menos favorecidas da população. Portanto, a instituição de políticas públicas de prevenção das DCVs deve ser intensificada com o objetivo de restituir a tendência de queda da mortalidade. Isto porque os fatores ambientais, ocupacionais, comportamentais e metabólicos foram responsáveis por quase $90 \%$ dos anos de vida ajustados por incapacidade e de mortes por DCVs (MANSUR; FAVARATO, 2016, p. 23-24).

\subsection{Hipertensão arterial}

Segunda a Sociedade Brasileira de Cardiologia, a pressão arterial elevada é um dos fatores de risco para doenças cardiovasculares. De acordo com a Organização Pan-americana da Saúde (2017, não paginado):

estima-se que 17,7 milhões de pessoas morreram por doenças cardiovasculares em 2015, representando 31\% de todas as mortes em nível global. Desses óbitos, estima-se que 7,4 milhões ocorrem devido às doenças cardiovasculares e 6,7 milhões devido a acidentes 
vasculares cerebrais (AVCs). Mais de três quartos das mortes por doenças cardiovasculares ocorrem em países de baixa e média renda.

A hipertensão arterial é uma doença circulatória. Diante do cenário, urge a necessidade de investigar e identificar a associação da hipertensão arterial com a elevação do risco para doenças cardiovasculares.

Segundo Whelton e outros autores (2012, p. 2881): "Um desafio no estudo do impacto da ingestão excessiva de sódio nos humanos é que os danos nos órgãosalvo muitas vezes se desenvolvem lentamente, provavelmente, durante muitos anos." ${ }^{\prime 4}$.

\section{2 Álcool, drogas e tabaco}

O uso do álcool, drogas e tabaco entre estudantes da educação básica é uma preocupação crescente. Agrava-se quando estes hábitos estão presentes no âmbito familiar.

O SISNAD, Sistema Nacional de Políticas Públicas sobre Drogas, tem como um de seus objetivos integrar as políticas de prevenção do uso indevido prevista na Lei no 11.343, de 23 de agosto de 2006 (BRASIL, 2006).

Entende-se que esta integração e engajamento deve se estender aos segmentos escolares, como prevenção, já desde o ensino fundamental, por meio de ações educativas, que dentre outras ações, tem a de levar o conhecimento sobre as drogas e a gravidade de sua utilização.

O Programa Nacional de Controle do Tabagismo, PNCT, é composto por ações articuladas através do Instituto Nacional de Combate ao Câncer ([2018]), INCA, junto à Secretarias de Educação e de Saúde. As ações são multiplicadas entre profissionais atuantes em escolas, dentre outras instituições envolvidas com o controle do tabagismo e prevenção de câncer.

O Programa tem como objetivo reduzir a prevalência de fumantes e a consequente morbimortalidade relacionada ao consumo de derivados do tabaco,

\footnotetext{
${ }^{4}$ Texto original: "A challenge in studying the impact of excess sodium intake in humans is that targetorgan damage often develops slowly, likely over many years".
} 
seguindo um modelo no qual ações educativas, de comunicação, de atenção à saúde, associadas às medidas legislativas e econômicas, se potencializam para prevenir a iniciação do tabagismo, promover a cessação de fumar e proteger a população da exposição à fumaça ambiental do tabaco. De iniciativa da Secretaria Nacional Antidrogas, há um curso de capacitação direcionado aos conselheiros municipais e lideranças comunitárias, Prevenção ao Uso Indevido de Drogas (BRASIL, 2011).

Segundo Costa e outros autores (2017, p. 206), o tabagismo somado a outro fator de risco, durante a adolescência, já é suficiente para a predição de uma doença cardiovascular nos próximos 10 anos, pois unidos elevam a gravidade das lesões vasculares, prevalecendo na fase adulta.

\subsection{Diabetes Mellitus}

Segundo a Sociedade Brasileira de Diabetes, atualmente, existem mais de 12 milhões de portadores da doença. Os diabéticos tipo 2 (DM2) representam 90\% a 95\% dos casos, sendo que a grande maioria apresenta sobrepeso ou obesidade. Apesar de ocorrer em qualquer idade, geralmente o DM2 é diagnosticado após os 40 anos. É importante ressaltar que o diabetes apresenta alta morbimortalidade, sendo uma das principais causas de mortalidade, insuficiência renal, amputação de membros inferiores, cegueira e de doença cardiovascular, incluindo doenças coronarianas e acidentes vasculares encefálicos.

[...] nos últimos anos tem se verificado um aumento da prevalência desta doença em crianças e adolescentes. Neste sentido, deve-se enfatizar que o diabetes tipo 2 tem contribuído com mais de $30 \%$ dos novos casos de diabetes, mostrando uma possível relação do aumento da prevalência de obesidade infantil com o desenvolvimento desta doença. Um estudo multicêntrico em 55 crianças e 112 adolescentes obesos constatou a existência da diminuição da tolerância à glicose em $25 \%$ e $21 \%$, respectivamente, sendo que $4 \%$ dos adolescentes eram diabéticos tipo 2. 0 índice de resistência à insulina foi um forte preditor para a diminuição da tolerância à glicose, confirmando que na infância, a resistência à insulina associada com a hiperinsulinemia são os fatores de risco mais importantes para o desenvolvimento da diminuição da tolerância à 
glicose em crianças obesas. O processo do desenvolvimento do diabetes tipo 2 na infância parece evoluir de maneira mais rápida do que nos adultos (OLIVEIRA et al., 2004, p. 239).

Embora seja reconhecido o impacto negativo do diabetes no cotidiano de seus portadores, bem como a presença de diversas variáveis envolvidas nesse processo, são escassas as pesquisas que revelem quais fatores da doença têm influenciado a qualidade de vida dos indivíduos diabéticos. Assim, são oportunos os estudos que busquem identificar essa relação, diante da importância que o Diabetes Melitus tem assumido no cenário da saúde pública mundial.

\section{DOENÇAS CARDIOVASCULARES}

As doenças cardiovasculares, para Carlucci (2013, p. 376), "são doenças que causam distúrbios no coração e vasos sanguíneos, responsáveis pela maior taxa de morbidade e mortalidade no mundo, sendo que requerem os mais elevados custos de assistência social e econômica".

Segundo Gagliardi (2009, p. 1), dentre as doenças cardiovasculares, o acidente vascular cerebral (AVC) é a principal causa de óbito e de incapacidade no Brasil, o que causa um alto custo para família e para o governo, que tem seu orçamento comprometido com o tratamento clínico e de reabilitação, e não tendo acesso às suas necessidades básicas.

O acidente vascular cerebral é frequente em adultos e é a segunda causa de morte no mundo e a primeira causa de incapacidade funcional para as atividades de vida diária. Segunda a Organização Mundial de Saúde (OMS), 15 milhões de pessoas apresentam acidente vascular cerebral por ano, destas cinco milhões morrem em decorrência do evento e grande parte dos sobreviventes apresenta sequelas físicas e ou mentais. As sequelas geram impacto econômico, social e familiar, sendo que $15 \%$ dos pacientes não apresentam prejuízo da capacidade funcional (RANGEL; BELASCO; DICCINI, 2013, p. 206).

Diante deste quadro, ratifica-se a importância das ações educativas no âmbito escolar, pelo tema Educação Alimentar e Nutricional, recentemente, incluído no currículo do ensino fundamental II e médio, da educação básica. Assim sendo, 
por meio do tema transversal, incluir as informações, os dados estatísticos das doenças cardiovasculares, os efeitos negativos sobre as pessoas que adquirem sequelas, as dificuldades na condução da vida diária, a saída do mercado de trabalho e o impacto na rotina familiar, de forma que os escolares entendam que podem evitar, em grande parte, estes infortúnios futuros, modificando seus hábitos atuais.

\section{METODOLOGIA}

O presente levantamento de informações sobre as matérias referentes às comorbidades e doenças cardiovasculares, se deu através de revisão bibliográfica, aplicado o método de pesquisa exploratória.

O estudo fundamentou-se em documentos de governo e de entidades ligadas à temática educação alimentar e nutricional, sendo a obesidade abordada como uma questão de política pública, presente nas áreas da educação e da saúde. Foram incluídos normativos específicos da alimentação escolar.

Foram pesquisados os sítios eletrônicos do Ministério da Educação (http:// portal.mec.gov.br), da Biblioteca Virtual do Ministério da Saúde (http://bvsms. saude.gov.br) e de órgãos vinculados como INEP - Instituto Nacional de Estudos e Pesquisas Educacionais Anísio Teixeira, CNE - Conselho Nacional de Educação e FNDE - Fundo Nacional de Desenvolvimento da Educação.

Foram acessadas as plataformas Scielo, Scopus e Pubmed, utilizando-se os descritores, doenças cardiovasculares, obesidade infantil, pressão arterial, sedentarismo e diabetes. Aplicando-se os filtros, dentre estes, artigos científicos foram escolhidos para compor a presente revisão.

\section{III FÓRUM DE MONITORAMENTO DAS METAS NACIONAIS E GLOBAIS PARA O ENFRENTAMENTO DAS DOENÇAS CRÔNICAS NÃO TRANSMISSÍVEIS NO BRASIL 2011-2022}

Segundo o Departamento de Vigilância de Doenças e Agravos Não Transmissíveis e Promoção da Saúde, a vigilância, monitoramento e avaliação das DCNT e respectivos fatores de risco são executados utilizando-se de inquéritos 
domiciliares, escolares, telefônicos e das informações do sistema de saúde. (BRASIL, 2018b, p. 25).

No que se refere à saúde dos escolares, em 2009, foi realizada a primeira edição da Pesquisa Nacional de Saúde do Escolar, com adolescentes das 26 capitais brasileiras e do Distrito Federal. Essa pesquisa é realizada a cada três anos e, em 2012, passou a ser realizada também nos Municípios do interior, além dos Municípios das capitais do Brasil. (BRASIL, 2018b, p. 26).

\section{OBJETIVOS}

O Censo Escolar é um levantamento de dados estatísticos educacionais anual e de âmbito nacional, determinado pelo Decreto $n^{0}$ 6.425, de 4 de abril de 2008, que em seu artigo 4 estabelece a obrigatoriedade do fornecimento de informações solicitadas no Censo Escolar da Educação Básica para todas as escolas públicas e privadas do país, constituindo-se numa ferramenta imprescindível para todos os atores na área de educação e sociedade em geral (BRASIL, 2008). O Censo é realizado pelo Instituto Nacional de Estudos e Pesquisas Educacionais Anísio Teixeira - INEP.

Segundo o INEP, os Indicadores Educacionais do Censo Escolar demonstram o desempenho dos alunos, o contexto socioeconômico e as condições em que se dá o processo de ensino e aprendizagem. Os Indicadores são úteis principalmente para o monitoramento dos sistemas educacionais, considerando o acesso, a permanência e a aprendizagem de todos os alunos. Contribuem para o acompanhamento de políticas públicas direcionadas para a melhoria da qualidade da educação e dos serviços oferecidos à sociedade (BRASIL, 2018c).

A Pesquisa Nacional por Amostra de Domicílios Contínua 2016-2017 realizada pelo IBGE, com divulgação em 2018, mostra que a taxa de escolarização, em 2017, para as pessoas de 6 a 14 anos de idade, foi 99,2\%, o que equivaleu a 26,2 milhões de estudantes, no sistema de ensino brasileiro. Em relação, às pessoas na faixa etária de 15 a 17 anos de idade, a taxa de escolarização, em 2017, foi de 87,2\%, 
todavia a equivalência em números dos estudantes não foi informada (INSTITUTO BRASILEIRO DE GEOGRAFIA E ESTATÍSTICA, 2018, p. 6).

Portanto, levando-se em conta a taxa de escolarização, o universo é estimado em milhões de estudantes, constatação suficiente para nortear o objetivo geral desta pesquisa.

No presente estudo, a indicação da faixa etária de 9 a 17 anos é justificada pelo grau de compreensão por parte dos estudantes e da autonomia em suas preferências alimentares.

O objetivo geral é a criação de políticas públicas visando a educação em saúde de forma mais abrangente. Há a necessidade de incluir nos regimentos internos escolares, que possuem forma normativa, regras específicas para atendimento dos escolares portadores de obesidade e de doenças decorrentes. Assim como, a previsão de encaminhamento às unidades de saúde para o devido acompanhamento. Esta parceria visa a redução de internações e da mortalidade infantil por causas preveníveis, na fase infantojuvenil e, consequentemente, o acometimento das doenças cardiovasculares, na idade jovem e adulta.

Os objetivos específicos traduzem-se em ações para responder de forma regular e estruturada, integrando ações preventivas em atenção aos alunos, com base em valores éticos e sociais, de forma a respeitar e contemplar as diferentes peculiaridades de cada região, local, cultura e condição socioeconômica, na temática da educação alimentar e nutricional.

Esta nova forma de organização em prol da educação alimentar como fator fundamental da prevenção de doenças, acredita-se ser de menor custo e de maior eficiência, comparada aos custos elevados do sistema de saúde.

A proposta e suas respectivas ações, nas escolas, objetivam o aumento da integração e do engajamento dos alunos, em colocar em prática os ensinamentos e orientações oferecidas pelos atores educacionais e de saúde envolvidos.

As escolas são coletivas e, portanto, entende-se que estas ações precisam ser montadas em uma linha estruturada de atuação, de forma a contemplar as individualidades dos alunos, suas experiências e emoções e desenvolver habilidades para que sejam indivíduos solidários, ao longo do percurso escolar. Deve-se 
considerar as dificuldades para a implementação e o acompanhamento por equipes de coordenadores.

\section{EDUCAÇÃO ALIMENTAR E NUTRICIONAL}

A educação alimentar e nutricional foi nominada como tema transversal e carece da compreensão abrangente necessária de diferentes saberes. A transversalidade diz respeito ao aprendizado de conhecimentos já sistematizados com os aprendidos na realidade do cotidiano.

"[...] por meio da articulação das diversas áreas do conhecimento, [...] se apresenta a possibilidade de uma nova organização curricular, que permita [...] introduzir novos arranjos de conteúdo, com base na interdisciplinaridade e temas transversais." (CARVALHO, 2012, p. 54).

Há que se afirmar a interdisciplinaridade como um conceito, historicamente e socialmente, produzido, apresentando no campo epistemológico, no mundo do trabalho, e na educação, movimento de continuidade e ruptura em relação às questões que buscam elucidar, e que, simultaneamente, a constituem. (PEREIRA, 2008, p. 263)

A melhor alimentação é aquela que reúne os nutrientes necessários e em quantidades suficientes para nutrir o organismo, em suas necessidades diárias. Uma dieta equilibrada traz vários benefícios aos estudantes: melhoria no rendimento escolar; desenvolvimento físico e psíquico; aumento das defesas orgânicas necessárias a boa saúde; redução do absenteísmo; alteração dos hábitos alimentares; aumento da disposição física para a prática de exercícios; elevação da autoestima pelo sentimento de pertencimento à coletividade.

O Objetivo de Desenvolvimento Sustentável (ODS) de número 3, da Agenda 2030, recomenda aos governos "assegurar uma vida saudável e promover o bemestar para todos, em todas as idades." (ORGANIZAÇÃO DAS NAÇÕES UNIDAS, 2015, não paginado).

\section{CONSIDERAÇÕES FINAIS}


As políticas públicas promoverão a integração e comprometimento dos profissionais envolvidos nas ações educativas e de promoção à saúde. São imprescindíveis para a comunicação e o fortalecimento de todas as medidas e diretrizes das áreas de educação e saúde. A interdisciplinaridade promoverá as transformações desejadas, por meio de ações prevencionistas, com o intuito de reduzir o acometimento da obesidade e suas consequências, nas faixas etárias mais jovens, como também, a redução de doenças cardiovasculares na fase precoce da vida e na fase adulta.

Estratégias coletivas poderão ser desenvolvidas como integrar atividades físicas e de recreação nas escolas e ofertar lanches e refeições saudáveis. É importante lembrar que as atividades físicas requerem profissionais qualificados e monitoramento adequado, com a preocupação de perceber a receptividade por parte dos alunos com excesso de peso e obesos, na lida com as práticas físicas.

\section{REFERÊNCIAS}

BIDDLE, S. J.; BENGOECHEA, E. G.; WIESNER, G. Sedentary Behaviour and Adiposity in Youth: A Systematic Review of Reviews and Analysis of Causality. International Journal of Behavioral Nutrition and Physical Activity, [S.I.], v. 14, n. 43, p. 121, 2017. Disponível em: https://ijbnpa.biomedcentral.com/articles/10.1186/s12966017-0497-8. Acesso em: $10 \mathrm{dez} .2018$.

BRASIL. Constituição da República Federativa do Brasil, 1988. Brasília, DF: Presidência da República, 1988. Disponível em: http://www.planalto.gov.br/ccivil_03/constituicao/constituicao.htm. Acesso em 18 out. 2018.

BRASIL. Decreto $\mathbf{n}^{0} \mathbf{6 . 4 2 5}$, de 4 de abril de 2008. Dispõe sobre o censo anual da educação. Brasília, DF: Presidência da República, 2008. Disponível em: http://www.planalto.gov.br/ccivil_03/_Ato2007-2010/2008/Decreto/D6425.htm. Acesso em: 05 dez. 2018.

BRASIL. Lei no 8.069, de 13 de julho de 1990. Dispõe sobre o Estatuto da Criança e do Adolescente. Brasília, DF: Presidência da República, 1990. Disponível em: http://www.planalto.gov.br/ccivil_03/leis//8069.htm. Acesso em: 05 dez. 2018.

BRASIL. Lei no 9.394, de 20 de dezembro de 1996. Estabelece as diretrizes e bases da educação nacional. Brasília, DF: Presidência da República, 1996. Disponível em: http://www.planalto.gov.br/ccivil_03/leis/19394.htm. Acesso em: 08 dez. 2018. 
BRASIL. Lei no 11.343, de 23 de agosto de 2006. Institui o Sistema Nacional de Políticas Públicas sobre Drogas - SISNAD. Brasília, DF: Presidência da República, 2006. Disponível em: http://www.planalto.gov.br/ccivil_03/leis/19394.htm. Acesso em: 08 dez. 2018.

BRASIL. Lei no 11.947, de 16 de junho de 2009. Dispõe sobre o atendimento da alimentação escolar. Brasília, DF: Presidência da República, 2009. Disponível em: http://www.planalto.gov.br/ccivil_03/_ato2007-2010/2009/lei/l11947.htm. Acesso em: 10 nov. 2018.

BRASIL. Lei no 13.666, de 16 de maio de 2018. Altera a Lei no 9.394, de 20 de dezembro de 1996, para incluir o tema transversal da educação alimentar e nutricional no currículo escolar. Brasília, DF: Presidência da República, 2018a. Disponível em: http://www.planalto.gov.br/ccivil_03/_Ato20152018/2018/Lei/L13666.htm. Acesso em: 10 dez. 2018.

BRASIL. Câmara dos Deputados. Projeto de Lei da Câmara no 102, de 2017. Inclui o tema da educação alimentar e nutricional nos currículos do ensino fundamental e do ensino médio. Brasília, DF: Senado Federal, 2017. Disponível em: https://www25.senado.leg.br/web/atividade/materias/-/materia/130899/pdf. Acesso em: 2 dez. 2018.

BRASIL. Ministério da Educação. Instituto Nacional de Estudos e Pesquisas Educacionais Anísio Teixeira. Censo Escolar 2017: notas estatísticas. Brasília, DF: MEC; INEP, 2018c. Disponível em: http://download.inep.gov.br/educacao_basica/censo _escolar/notas_estatisticas/2018/notas_estatisticas_Censo_Escolar_2017.pdf. Acesso em: 15 nov. 2018.

BRASIL. Ministério da Educação. Diretrizes curriculares nacionais para educação básica. Brasília: MEC, 2013a.Disponível em: http://portal.mec.gov.br/docman/julho-2013-pdf/13677-diretrizes-educacao-basica2013-pdf/file. Acesso em: 06 dez. 2018.

BRASIL. Ministério da Educação. Resolução FNDE no 26, de 17 de junho de 2013. Dispõe sobre 0 atendimento da alimentação escolar aos alunos da educação básica no âmbito do PNAE. Brasília, DF: MEC, 2013b. Disponível em: https://www.fnde.gov.br/acesso-a-informacao/institucional/legislacao/item/4620resolu\%C3\%A7\%C3\%A3o-cd-fnde-n\%C2\%BA-26,-de-17-de-junho-de-2013. Acesso em: 5 dez. 2018.

BRASIL. Ministério da Justiça. Secretaria Nacional de Políticas sobre Drogas. Prevenção ao uso indevido de drogas: capacitação para conselheiros e lideranças comunitárias. Brasília, DF: Ministério da Justiça; SENAD, 2011. Disponível 
em:

http://www.mppr.mp.br/arquivos/File/Projeto_Semear/Material_Capacitacao/Curso_P revencao_ao_uso_indevido_de_Drogas_Capacitacao_para_Conselheiros_e_Lideranca s_Comunitarias_2011_SENAD.pdf. Acesso em: 17 nov. 2018.

BRASIL. Ministério da Saúde. Portaria no 2.446, de 11 de novembro de 2014. Redefine a Política Nacional de Promoção da Saúde (PNPS). Brasília, DF: Ministério da Saúde, 2014a. Disponível em:

http://bvsms.saude.gov.br/bvs/saudelegis/gm/2014/prt2446_11_11_2014.html. Acesso em: 11 dez. 2018.

BRASIL. Ministério da Saúde. Secretaria de Atenção à Saúde. Estratégias para o cuidado da pessoa com doença crônica: obesidade. Brasília, DF: Ministério da Saúde, 2014b. (Cadernos de Atenção Básica, n. 38). Disponível em: http://bvsms.saude.gov.br/bvs/publicacoes/estrategias_cuidado_doenca_cronica_obe sidade_cab38.pdf. Acesso em: 13 nov. 2018.

BRASIL. Ministério da Saúde. Secretaria de Vigilância em Saúde. Relatório do III Fórum de Monitoramento do Plano de Ações Estratégicas para o Enfrentamento das Doenças Crônicas não Transmissíveis no Brasil. Brasília, DF:

Ministério da Saúde, 2018b. Disponível em:

http://bvsms.saude.gov.br/bvs/publicacoes/relatorio_iii_forum_monitoramento_plano .pdf. Acesso em 11 dez de 2018.

CARLUCCI, E. M. S. et al. Obesidade e sedentarismo: fatores de risco para doença cardiovascular. Com. Ciências Saúde, Brasília, DF, v. 24, n. 4, p. 375-384, 2013. Disponível em:

http://bvsms.saude.gov.br/bvs/artigos/ccs/obesidade_sedentarismo_fatores_risco_ca rdiovascular.pdf. Acesso em: 30 nov. 2018.

CARVALHO, E. J. G. Gestão escolar: da centralização à descentralização. Cadernos de Pesquisa em Educação PPGE/UFES, Vitória, ano 9, v. 18, n. 36, p. 33-53, 2012. Disponível em:

http://www.periodicos.ufes.br/educacao/article/view/5373/3973. Acesso em: 7 dez. 2018.

COELHO, L. G. et al. Associação entre estado nutricional, hábitos alimentares e nível de atividade física em escolares. Jornal da Pediatria, Rio de Janeiro, v. 88, n. 5, p. 406-412, 2015. Disponível em: http://www.scielo.br/pdf/jped/v88n5/v88n05a09.pdf. Acesso em: 10 dez. 2018.

COSTA, I. F. A. F. et al. Adolescentes: comportamento e risco cardiovascular. Jornal Vascular Brasileiro, Porto Alegre, v. 15, n. 3, p. 205-213, 2017. Disponível em: 
http://www.scielo.br/pdf/jvb/v16n3/1677-5449-jvb-1677-5449011816.pdf. Acesso em: 8 nov. 2018.

DIAS, P. C. et al. Obesidade e políticas públicas: concepções e estratégias adotadas pelo governo brasileiro. Cadernos de Saúde Pública, Rio de Janeiro, v. 33, n. 7, p. 1-12, 2017. Disponível em: http://www.scielo.br/pdf/csp/v33n7/1678-4464-csp-3307-e00006016.pdf. Acesso em: 28 out. 2018.

FREITAS, M. C. S. et al. Escola: lugar de estudar e de comer. Ciência \& Saúde Coletiva, Rio de Janeiro, v. 18, n. 4, p. 979-985, 2013. Disponível em: http://www.scielo.br/pdf/csc/v18n4/10.pdf. Acesso em: 12 dez. 2018.

ECKEL, R. H. et al. Guideline on Lifestyle Management to Reduce Cardiovascular Risk. AHA/ACC, Dallas, v. 129, n. 25, suppl. 2, p. S76-S99, 22013 Disponível em: https://www.ahajournals.org/doi/pdf/10.1161 /01.cir.0000437740.48606.d1. Acesso em: 7 nov. 2018.

GAGLIARDI, R. J. Hipertensão arterial e AVC. Com Ciência, Campinas, n. 109, 2009. Disponível em: http://comciencia.scielo.br/pdf/cci/n109/a18n109.pdf. Acesso: em 21 nov. 2018.

INSTITUTO BRASILEIRO DE GEOGRAFIA E ESTATÍSTICA. Pesquisa Nacional por Amostra de Domicílios (PNAD) Contínua 2016-2017. [Rio de Janeiro]: IBGE, 2018. p. 1-12. Disponível em:

https://biblioteca.ibge.gov.br/visualizacao/livros/liv101576informativo.pdf. Acesso em: 06 dez. 2018.

Instituto Nacional do Câncer. Ministério da Saúde. Programa Nacional de Controle do Tabagismo. [S.I.]: INCA, [2018]. Disponível em:

https://www.inca.gov.br/programa-nacional-de-controle-do-tabagismo. Acesso em: 7 nov. 2018.

LUZ, M. T. Saúde. In: PEREIRA, I. B.; LIMA, J. C. F. (org.). Dicionário da

Educação profissional em saúde. 2. ed. rev. e ampl. Rio de Janeiro: EPSJV, 2008. Disponível em: http://www.sites.epsjv.fiocruz.br/dicionario/Dicionario2.pdf. Acesso em: 07 dez. 2018.

MANSUR, A. P., FAVARATO, D. Tendências da Taxa de Mortalidade por Doenças Cardiovasculares no Brasil, 1980-2012. Arq Bras Cardiol, São Paulo, v. 107, n. 1, p. 20-25, 2016. Disponível em: http://www.scielo.br/pdf/abc/v107n1/pt_0066-782Xabc-20160077.pdf. Acesso em: 4 nov. 2018.

OLIVEIRA, A. M. A. et al. Sobrepeso e obesidade infantil: influência de fatores biológicos e ambientais em Feira de Santana, BA. Arquivos Brasileiros de Endocrinologia e Metabologia, São Paulo, v. 47 n. 2, p. 144-150, 2003. 
Disponível em: http://www.scielo.br/pdf/abem/v47n2/a06v47n2.pdf. Acesso em: 7 dez. 2018.

OLIVEIRA, C. L. et al. Obesidade e síndrome metabólica na infância e na adolescência. Revista de Nutrição, Campinas, v. 17, n. 2, p .237-245, 2004. Disponível em: http://www.scielo.br/scielo.php?pid=S141552732004000200010\&script=sci_abstract\&tlng=pt. Acesso em: 4 out. 2018.

Organização das Nações Unidas. Transformando nosso mundo: A Agenda 2030 para o Desenvolvimento Sustentável. Tradução: Centro de Informação das Nações Unidas para o Brasil. Rio de Janeiro: UNIC, 13 out. 2015. Disponível em: https://nacoesunidas.org/pos2015/agenda2030/. Acesso: 10 dez. 2018.

ORGANIZAÇÃO PAN-AMERICANA DA SAÚDE. Doenças cardiovasculares. Brasília, DF: OMS Brasil, 2017. Disponível em:

https://www.paho.org/bra/index.php?option=com_content\&view=article\&id=5253:d oencas-cardiovasculares\&Itemid=1096. Acesso em: 7 dez. 2018.

PEREIRA, I. B. Interdisciplinaridade. In: PEREIRA, I. B.; LIMA, J. C. F. (org.). Dicionário da Educação profissional em saúde. 2. ed. rev. e ampl. Rio de Janeiro: EPSJV, 2008. p. 263-268. Disponível em:

http://www.epsjv.fiocruz.br/sites/default/files/l43.pdf. Acesso em: 07 dez. 2018.

POLSKY, V. A. S. et al. Obesidade: epidemiologia, etiologia e Prevenção. In:

Nutrição Moderna de Shils na Saúde e na Doença. 11. ed. Barueri: Manoele, 2016.

RANGEL, E. S. S.; BELASCO, A. G. S.; DICCINI, S. Qualidade de vida de pacientes com acidente vascular cerebral em reabilitação. Acta Paulista de Enfermagem, São Paulo, v. 26, n. 2, p. 205-2012. Disponível em: http://www.scielo.br/pdf/ape/v26n2/v26n2a16.pdf. Acesso em: 06 dez. 2018.

WHELTON, P. K. et al. Sodium, blood pressure, and cardiovascular disease further evidence supporting the American Heart Association sodium reduction recommendations. AHA Journals, Dallas, v. 126, n. 24, p. 2880-2889, 2012. Disponível em: https://www.ahajournals.org/doi/10.1161/cir.0b013e318279acbf. Acesso em: 7 dez. 2018.

WORLD HEALTH ORGANIZATION. ICD-11 for Mortality and Morbidity Statistics: 2018 version. [S.I.]: Who, 2018. Disponível em: https://icd.who.int/browse11/l-m/en. Acesso em: 11 dez. 2018. 